\title{
Spitzer Survey of the Large Magellanic Cloud: Surveying the Agents of a Galaxy's Evolution (SAGE)
}

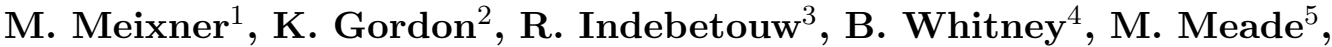 \\ B. Babler ${ }^{5}$, J. Hora ${ }^{6}$, U. Vijh ${ }^{1}$, S. Srinivasan ${ }^{1}$, C. Leitherer ${ }^{1}$, \\ M. Sewilo ${ }^{5}$, C. Engelbracht ${ }^{2}$, M. Block ${ }^{2}$, B. For ${ }^{7}$, R. Blum ${ }^{8}$, \\ W. Reach ${ }^{9}$, J-P. Bernard ${ }^{10}$ and the SAGE Team \\ ${ }^{1}$ Space Telescope Science Institute, 3700 San Martin Dr., Baltimore, MD 21218, USA \\ email: meixner@stsci.edu \\ ${ }^{2}$ University of Arizona, ${ }^{3}$ University of Virgina, ${ }^{4}$ Space Sciences Institute, ${ }^{5}$ University of \\ Wisconsin, ${ }^{6}$ Harvard/CfA, ${ }^{7}$ University of Texas, ${ }^{8} \mathrm{NOAO} /$ Tucson, ${ }^{9}$ Spitzer Science Center, \\ ${ }^{10} \mathrm{CESR}$
}

\section{Summary}

We are performing a uniform and unbiased imaging survey of the Large Magellanic Cloud (LMC), using the IRAC and MIPS instruments on board the Spitzer Space Telescope (Spitzer). Meixner et al. (2006) provides an overview of the project and initial results and their Table 1 (repeated here) outlines the survey's salient characteristics. In this project, we are surveying the agents of a galaxys evolution (SAGE), i.e. the interstellar medium (ISM) and stars, and their interaction on the galaxy wide scale of the LMC. Spitzer IRAC and MIPS images provide key insights into the life cycle of matter in a galaxy because the infrared emission from dust grains is an effective tracer of the ISM, star formation, and stellar mass-loss. Three key science goals determined the coverage and depth of the survey. The detection of diffuse ISM with column densities $>1.2 \times 10^{21}$ $\mathrm{H} \mathrm{cm}^{-2}$ permits detailed studies of dust processes in the ISM. SAGE's point source sensitivity enables a complete census of newly formed stars with masses $>3 \mathrm{M}_{\odot}$ that will determine the current star formation rate in the LMC. SAGE's detection of evolved stars with mass loss rates $>10^{-8} \mathrm{M}_{\odot} \mathrm{yr}^{-1}$ will quantify the rate at which evolved stars inject mass into the ISM of the LMC (Blum et al. 2006). The SAGE data are nonproprietary. The preliminary SAGE catalog of epoch 1 photometry, prepared by the SAGE Team and released to the public on January 3, 2006, contains over 4 million IRAC sources, band merged with 2MASS photometry and over 60,000 MIPS 24 micron sources. Preliminary estimates indicate that foreground Milky Way stars and background galaxies may comprise as much as $18 \%$ and $12 \%$, respectively, of these catalogs. To learn more about the SAGE project: http://sage.stsci.edu/ . 

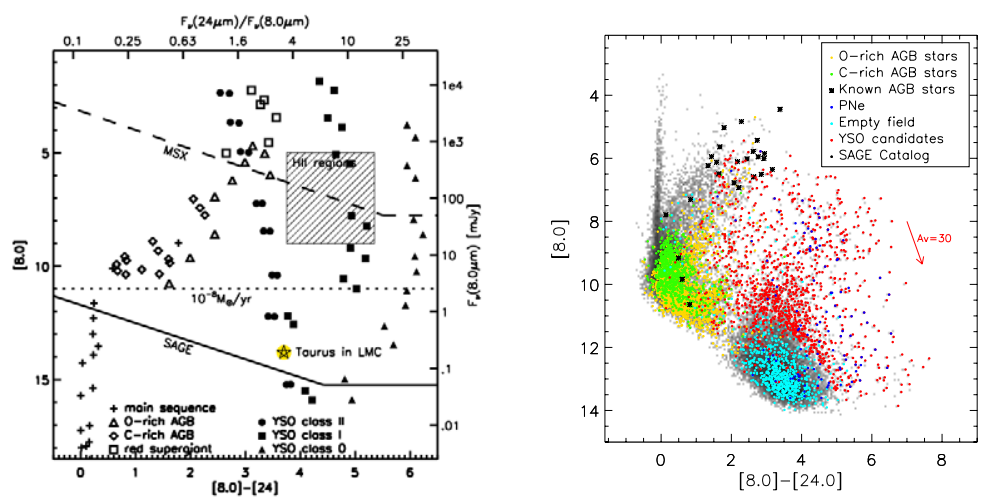

Figure 1. Left: The SAGE discovery space for LMC objects where [8.0] and [24] are the Vega magnitudes at 8 and $24 \mu \mathrm{m}$, respectively (Meixner et al. 2006). Right: Preliminary results on source classification based on the SAGE Epoch 1 catalogs. All SAGE sources in grey and classes overlaid in color as indicated in the legend.

Table 1. Principal Characteristics for SAGE Survey (Meixner et al. 2006)

\begin{tabular}{|c|c|c|}
\hline Characteristic & IRAC Value & MIPS Value \\
\hline \multicolumn{3}{|l|}{ Nominal Center point } \\
\hline $\mathrm{RA}(2000)$ & $5 \mathrm{~h} 18 \mathrm{~m} 48 \mathrm{~s}$ & $5 \mathrm{~h} 18 \mathrm{~m} 48 \mathrm{~s}$ \\
\hline $\operatorname{Dec}(2000)$ & $-68^{\circ} 34^{\prime} 12^{\prime \prime}$ & $-68^{\circ} 34^{\prime} 12^{\prime \prime}$ \\
\hline survey area & $7.1^{\circ} \times 7.1^{\circ}$ & $7.8^{\circ} \times 7.8^{\circ}$ \\
\hline AOR size, grid size & $1.1^{\circ} \times 1.1^{\circ}, 7 \times 7$ & $25^{\prime} \times 4^{\circ}, 19 \times 2$ \\
\hline Total time (hrs) & 290.65 & 216.84 \\
\hline$\lambda(\mu \mathrm{m})$ & $3.6,4.5,5.8$ and 8 & 24,70 and 160 \\
\hline pixel size at $\lambda$ & $1.2^{\prime \prime}, 1.2^{\prime \prime}, 1.2^{\prime \prime}, 1.2^{\prime \prime}$ & $1.2^{\prime \prime}, 4.8^{\prime \prime}, 15.6^{\prime \prime}$ \\
\hline angular resolution at $\lambda$ & $1.7^{\prime \prime}, 1.7^{\prime \prime}, 1.9^{\prime \prime}, 2^{\prime \prime}$ & $5.8^{\prime \prime}, 17^{\prime \prime}, 38^{\prime \prime}$ \\
\hline Exposure time/pixel at $\lambda(\mathrm{s})$ & $48,48,48,48$ & $60,30,9$ \\
\hline \multicolumn{3}{|l|}{$\begin{array}{l}\text { Predicted point source } \\
\text { sensitivity, } 5 \sigma \text { at }\end{array}$} \\
\hline$\lambda(\mathrm{mJy})$ & $0.0051,0.0072,0.041,0.044$ & $0.31,10,60$ \\
\hline \multicolumn{3}{|l|}{$\begin{array}{l}\text { Predicted point source } \\
\text { sensitivity } 5 \sigma \text { at }\end{array}$} \\
\hline$\lambda$ (mag.) & $19.3,18.5,16.1,15.4$ & $10.9,3.5,-0.6$ \\
\hline $\begin{array}{l}\text { Saturation limits } \\
(\mathrm{Jy}) \text { at } \lambda\end{array}$ & $1.1,1.1,7.4,4.0$ & $4.1,23,3$ \\
\hline $\begin{array}{l}\text { Saturation limits } \\
\text { (mag) at } \lambda \\
\text { Surface brightness }\end{array}$ & $6,5.5,3.0,3.0$ & $0.61,-3.7,-3.2$ \\
\hline limits $(\mathrm{MJy} / \mathrm{sr})$ at $\lambda$ & $\ldots, \ldots, 0.5,1$ & $1,5,10$ \\
\hline Epoch 1 & July $15-26,2005$ & July 27 - Aug. 3, 2005 \\
\hline Epoch 2 & Oct. 26 - Nov. 2, 2005 & Nov. 2-9, 2005 \\
\hline
\end{tabular}

\section{Acknowledgements}

SAGE is supported by NASA/Spitzer grant 1275598 and NASA NAG5-12595.

\section{References}

Meixner, M., Gordon, K., Indebetouw, R. et al. 2006, AJ 132, 2268

Blum, B., Mould, J.R., Olsen, K.A. et al. 2006, AJ 132, 2034 